\title{
Egocentric Projection is a Rational Strategy for Accurate Emotion Prediction
}

\author{
Zidong Zhao*, Diana I. Tamir
}

Department of Psychology, Princeton University, Princeton, NJ08540

Word count: 8336

* Address correspondence to:

Zidong Zhao

Department of Psychology

Princeton University

Peretsman Scully Hall

Princeton, NJ 08540

USA

zidong@princeton.edu 


\begin{abstract}
People need to accurately understand and predict others' emotions in order to build and maintain meaningful social connections. However, when they encounter new social partners, people often do not have enough information about them to make accurate inferences. Rather, they often resort to an egocentric heuristic, and make predictions about a target by using their own self-knowledge as a proxy. Is this egocentric heuristic a form of cognitive bias, or is it a rational strategy for real-world social prediction? If egocentrism provides a rational and effective solution to the challenging task of social prediction in naturalistic contexts, we should expect that a) egocentric predictions tend to be more accurate, and b) people rely on self-knowledge to a greater extent when it's more likely to be a good proxy. Using an emotion prediction task and personality measures, we assessed similarity and predictive accuracy between first year college students and their new acquaintance roommate. Results demonstrated that, when people need to make predict an unfamiliar target's emotions, self-knowledge can often effectively approximate knowledge about others, and thus support accurate predictions. Moreover, participants that were typical of the sample, whose self-knowledge can better approximate information about the target, relied more on self-knowledge in their predictions, and thus achieved higher accuracy. These findings suggest that people rationally tune their use of egocentrism based on whether it is likely to pay off. Overall, these findings demonstrate a rational side to a cognitive phenomenon usually framed as a cognitive pitfall, namely egocentric projection, when its natural decision context is taken into consideration.
\end{abstract}




\section{Egocentric Projection is a Rational Strategy for Accurate Emotion Prediction}

To have positive, successful social interactions, people must accomplish the key task of social prediction. They must anticipate how others might think, feel, and act in the future, and ready themselves accordingly. The ability to see into the social future is particularly important when people are trying to establish new interpersonal connections. The stakes are high with new social targets, as first impressions are influential and long lasting (Rosenbaum, 1986). Moreover, the task itself is more challenging. When making predictions about new acquaintances, people have no shared history or accumulated person-specific knowledge to turn to. Instead, they must rely on other social prediction strategies. How do people resolve the challenge of social prediction, even in the absence of rich knowledge about the target?

Egocentrism - anchoring social inferences on self-knowledge - is one default strategy in the social prediction toolkit. It is a heuristic solution that is often seen as error prone. However, we propose that egocentrism is a rational decision strategy, for at least two reasons. First, we hypothesize that egocentrism generates accurate predictions. Second, we hypothesize that people deploy it strategically, based on how representative they are of their social environment. In two studies, we set out to test these interrelated ideas.

\section{Social prediction facilitates social connections}

People have a fundamental need to create meaningful social relationships (Baumeister \& Leary, 2017). Throughout their lives, people strive to connect with the people they encounter for collaboration, friendship, and romantic intimacy. To form these connections, it is crucial that people foster positive interaction with others, especially in the early stages of acquaintanceship.

One critical skill that enables successful social interactions is the ability to see into the social future (Tamir \& Thornton, 2018). That is, social perceivers need to anticipate how others 
might think, feel, and even act before these events take place. By doing so, people can prepare their own actions ahead of time, and thus make social interactions more seamless. Oftentimes, people are familiar with the targets of their social prediction. Through their experiences with familiar targets, people accrue rich, person-specific knowledge that aid them in making predictions (Stinson \& Ickes, 1992; Biesanz et al., 2007; Zhao et al., 2020). Information about the target's trait-like characteristics, as well as how they felt or acted in similar situations in the past, can help greatly in honing social predictions to be more accurate.

However, when people make social prediction about novel, unfamiliar targets, as is often the case when they attempt to build new connections, they do not have detailed person knowledge at their disposal. How do people resolve the complex challenge of social prediction when they're confronted with its full force?

One default heuristic used in social prediction is egocentrism (Epley et al., 2004). In egocentric inferences, people use their rich and easily accessible first-person knowledge - how they themselves might think or feel if they were in their target's situation - to approximate the possible experiences of the target. That is, people ignore a large number of potential cues about the target, thus relaxing the computational challenge of social prediction. Evidence of egocentrism abounds across many domains of social judgment. When asked what others notice or pay attention to, people routinely base their judgments on what is salient to themselves, ascribing their own attentional states to the target (Gilovich et al., 2000). Along a similar vein, when people are asked about others' knowledge state, they often anchor on what they themselves know. People even assume that private information, such as one's own feelings or intentions, is accessible to other people (Gilovich et al., 1998; Keysar, 1994; Keysar \& Henly, 2002). People rely heavily on self-knowledge extensively in their social judgments. 


\section{The rationality of egocentrism}

Much past research has regarded this egocentric strategy as an irrational misstep. For example, in the seminal work on the false consensus effect, participants routinely overestimated how much others shared their opinions and preferences, even when in the minority (Ross et al., 1977). Similarly, research has demonstrated that people tend to ascribe their own mental states to others more than is warranted, leading to the illusion of transparency or the curse of knowledge (Gilovich et al., 1998; Camerer et al., 1989; Birch \& Bloom, 2007). Research has also focused on the detrimental downstream consequences of egocentrism. For example, an undue sense of selfother agreement has been linked to negative outcomes such as mis-identifying attitudinal norms and undesirable behaviors (Bauman \& Geher, 2002), as well as having more polarized attitudes around social issues (Judd \& Johnson, 1981; Wojcieszak, 2008). Such a dominant bias framing masks the potential utility of egocentrism.

We suggest that egocentrism can, in fact, be considered a rational strategy for social prediction, for at least three reasons (Lieder \& Griffiths, 2020). First, egocentrism is statistically sound in certain circumstances. Specifically, egocentrism reflects a valid statistical procedure for making social predictions if knowledge about the self is the only available piece of evidence (Dawes, 1989). While self-knowledge constitutes a very small sample of all possible data points, it is procedurally rational to integrate this sample into one's prediction in the absence of other information (e.g., information about the target).

Second, egocentrism is effective. That is, self-knowledge can lead to accurate social inferences. Social judgment can be thought of as an optimization problem. To solve this problem, people use knowledge from different sources, such as knowledge about the self and about different social groups. The challenge is to find the most optimal way to weight and 
combine these sources of knowledge to make the most accurate judgment possible. How much weight on self-knowledge is optimal? One study calculated participants' optimal level of egocentrism (Hoch, 1987). In this study, participants predicted how much a social target would agree with a series of attitude statements (e.g., communism is the world's greatest peril), and reported their own attitudes for the same items. Analyses under this optimization framework showed that the majority of participants would have been more accurate had they been even more egocentric than they actually were. That is, self-knowledge is an effective proxy for knowledge about others, further supporting the value of egocentrism in social inferences.

Third, other sources of information that people rely on during social inference, such as stereotypes, can be inaccurate (Eyal et al., 2018; McConnell et al., 1994; Terracciano et al., 2005). In one series of studies, when participants were encouraged to abandon their egocentric defaults and actively engage in perspective taking by "putting themselves in others' shoes", the accuracy of their interpersonal judgments was found to decrease. These negative effects of perspective taking were attributed to the inaccuracy of participants' stereotypes about groups. This further highlights the potential importance of self-knowledge for accurate social judgment.

In the current study, we test the hypothesis that egocentrism is a rational strategy for social judgments in the domain of emotion prediction. We expect that the more people rely on self-knowledge to make social inferences, the more accurate they will be in their social predictions. Importantly, we test this in the context of real-world social inferences at the very start of new relationships.

\section{Similarity as a rational cue to be egocentric}

To make rational decisions, people must flexibly adapt their strategy based on relevant contextual cues (Griffiths, 2020; Griffiths et al., 2015). When contextual cues indicate that one 
strategy is more likely to lead to accurate judgment, people should be more likely to employ that strategy. One central cue for egocentrism in the domain of social prediction is dyadic similarity, or the similarity between a perceiver and the target of prediction. When people base their predictions on self-knowledge, their accuracy should increase, but only to the extent that this self-knowledge is also true of their target. Thus, perceivers who are similar to their target should experience the greatest benefit from egocentrism.

Indeed, research has shown that people do not employ an egocentric strategy in a onesize-fit-all fashion. Rather, people selectively engage in egocentrism only when it is most rational to do so: people are more likely to use self-knowledge to make an inference about a similar target than a dissimilar target (Robbins \& Krueger, 2005). The effect of similarity on this social inference strategy is both pervasive and profound. Egocentrism reliably increases with interpersonal similarity, including similarity in personality traits (Cho \& Knowles, 2013), academic and professional affiliations (Holtz, 2003; Ames, 2004a, 2004a), nationality (Li \& Hong, 2016), political orientation (Tamir \& Mitchell, 2013), and ethnicity (Granberg et al., 1981).

Similarity along one social dimension can affect how much people recruit self-knowledge to make judgments on other, potentially unrelated dimensions. For example, in Tamir and Mitchell (2013), participants engaged in more egocentric strategies when they judged a similar target's habits and preferences, even though target similarity was manipulated using political beliefs. In studies using a minimal group paradigm (Tajfel, 1970), researchers manipulate perceived similarity by dividing participants into categories using fictitious and uninformative criteria. This design removes any potential correspondence between the similarity dimension and the judgment dimension. Even so, people still employ self-knowledge in their inferences about 
minimal groups (Clement \& Krueger, 2002). People assume that those who are similar to them in some respect will also be similar to them in others, allowing perceived similarity to exert profound influence on social judgments.

If egocentrism leads to overall more accurate social judgments and people flexibly employ self-knowledge when the target is similar, people should make more accurate inferences about similar targets. Indeed, this prediction has been supported empirically. In one study, participants predicted the attitudes of different targets (Hoch, 1987). Those who made predictions about a more similar target (e.g., their spouse or an average graduate school peer) were more egocentric and more accurate than those who made predictions about a dissimilar target (e.g., the average American consumer). In a more recent study (Woo \& Mitchell, 2020), participants predicted social targets' attitudes and preferences. They similarly found that when people made predictions about targets that they considered more similar to themselves, their predictions were more egocentric and more accurate. In sum, people rationally adjust their egocentric strategy, ultimately leading to more accurate predictions about more similar others.

\section{Typicality as a rational cue to be egocentric}

Dyadic similarity provides a strong cue to use egocentric prediction. However, it might not be the most useful cue when people make predictions about a new, unfamiliar target. Dyadic similarity is variable and must be calculated on a case by case basis as people meet new targets. When engaging with a new person, people might not have enough time to comprehensively assess how alike they are. If people adjust their egocentric strategy to an unreliable sense of dyadic similarity, they might make less accurate social predictions.

Instead, we propose that interpersonal similarity informs social inference strategies over a broader timescale. People differ in how similar they tend to be, in general, to others in their 
immediate social environment. In other words, people are typical to varying extents. Much like similarity at the dyad level, a person's typicality influences the effectiveness of egocentrism. If a person is more typical, they are, by definition, more similar to more people in their social environment. If a person is similar to an individual target, they can make more accurate predictions by relying on self-knowledge in that specific instance. As such, typical people should, on average, make more accurate predictions by being egocentric. Over the course of people's social lives, typical people should gradually learn that they can make more accurate predictions about a novel target simply by defaulting to using themselves as an anchor. If people rationally adapt their inferential strategy in this way, we should see more typical people use more self-knowledge to make social prediction.

Prior work on typicality has primarily focused on the typicality of a perceived object, rather than the perceiver (Cantor \& Mischel, 1979; Eberhardt et al., 2006; Nosofsky, 1988; Rosch et al., 1976). Extant studies on the typicality of a perceiver have largely investigated the agent's own perceived typicality. For example, this work finds that a group members' own perceived typicality can predict how likely they will be to "go to the extreme" for the group (Goldman \& Hogg, 2016), or to align themselves with stigmatized groups (Essien et al., 2020). In the current study, we focus not on how typical one perceives themself to be. Rather, we focus on ground truth typicality - how typical people actually are in relation to their social surroundings. Self-knowledge can only be a valid cue for accurate social prediction to the extent that people are actually typical.

\section{The current studies}

Across two studies, we aimed to demonstrate and replicate, respectively, that (i) egocentrism is a rational strategy that leads to accurate predictions, and (ii) perceivers rationally 
adapt their egocentrism based on their own typicality and dyadic similarity. To do so, we investigate three sets of interrelated hypotheses:

First, we hypothesize that egocentrism is an overall effective strategy for social prediction. We expect that greater egocentrism should be associated with more accurate social predictions.

Second, we hypothesize that perceiver typicality is an informative cue for using egocentric strategies. We first replicate prior work showing that dyadic similarity leads people to rely on self-knowledge. We propose that individuals develop their social inference strategies over a lifetime, such that the typicality of a perceiver serves as the most informative cue for employing egocentric strategies. We expect that more typical perceivers should use more egocentric strategies.

Finally, we hypothesize that perceiver typicality improves how accurately people make predictions about novel individuals. Specifically, it does so by increasing the use of egocentric social inference strategies between the perceiver and their target. That is, we expect more typical perceivers will be more egocentric, which in turn leads to more accurate predictions.

\section{Code and data availability}

Data and code from Studies One and Two freely available on the Open Science Framework (https://osf.io/s7xzn/). Analyses conducted in Study 2 were preregistered. We report how we determined sample size, all data exclusions, all manipulations, and all measures in the study. All analyses were conducted using R (R core team, 2018). Mediation models were conducted using the 'lavaan' package(Rosseel, 2012), and graphics were generated using the 'ggplot2' package and the 'semPlot' package (Epskamp, 2015). All null hypothesis significance tests are two-tailed. 


\section{Study 1}

\section{Methods}

\section{Participants}

Participants were first-year undergraduate students at Princeton University. Each student signed up for the study with one roommate at the outset of the fall semester. Participants were recruited as the first wave of a longitudinal study, though subsequent waves are not analyzed as part of this investigation. A sample size of 40 dyads was set a priori, and 59 newly formed roommate dyads were recruited to allow for attrition $(\mathrm{N}=118,88$ women; Mean age $=18.44$, $\mathrm{SD}=1.65 ; 46.6 \%$ White, $33.9 \%$ Asian, $8.5 \%$ more than one ethnicity, $7.6 \%$ Black or African American, 1.7\% others, $0.8 \%$ Alaskan Native and American Indian, and 0.8\% unreported; 9.3\% Hispanic or Latinx).

All participants were recruited within six weeks of the first day of the semester, and all completed the study within that time frame (mean $=24$ days; range $=0-40$ days). Participants were assigned roommates by the university and had known each other for an average of 26.7 days (range $=0-83$ days). One dyad was excluded from analyses because of prior acquaintanceship (relationship duration $=345$ days). All participants provided informed consent in a manner approved by the Princeton University Institutional Review Board.

\section{Procedure}

Participants were recruited in pairs but instructed to complete the study online independently. During the study, participants first completed an emotion transition task in which they made predictions about their own emotion transitions as well as their partner's transitions. We used this task to measure how accurate participants were at emotion predictions, how similar they were to their target, and how typical they were as a member of the university community. 
Participants then completed questionnaires about four aspects of their personality: values, attitudes, recreational activities, and feelings towards different social groups. We used these personality questionnaires to assess their dyadic similarity and typicality outside the domain of emotion dynamics. Finally, participants completed measures of their relationship and general social wellbeing. These measures were administered as a component of the larger longitudinal projects and were not included in any current hypotheses or analyses.

Emotion Transition Task. In the emotion transition task, participants made a series of predictions about how a target's emotions might change over time. Specifically, participants judged how likely it was that one mental state (e.g., happiness) would transition into another (e.g., elation) for a particular target. On each trial, participants saw a target (e.g., self) and a pair of emotional states (e.g., happiness $\rightarrow$ elation). On a continuous scale from $0 \%$ to $100 \%$, participants rated the likelihood that the target would next experience the second mental state, given that they were currently experiencing the first.

Three independent sets of five emotion states were used to construct task stimuli. These states were selected by first applying k-medoids clustering to three large sets of transitional probability data from previous research (Thornton \& Tamir, 2017), and then taking the central state of each identified cluster. This selection procedure ensured that the states used in the current study covered the full span of the mental state space (See Supplement for details). Stimuli included all possible state pairs from within each set. Participants thus completed a total of 75 trials for a single target. Trials were randomized within target blocks.

Participants completed the task for three targets: (i) Self: a participant's ratings of their own emotion transitions, (ii) Target: a participant's ratings of their partner's emotion transitions, and (iii) Group: a participant's ratings of the emotion transitions of the average student at their 
university. The order of targets was randomized across participants. For the purpose of the present study, only Self ratings and Target ratings were analyzed.

We used the emotion transition ratings to assess four constructs of central interest: accuracy, egocentrism, similarity, and typicality. Accuracy refers to how well one's predictions about their target match the target's actual emotion transitions. To assess a participant's accuracy, we computed the Pearson's correlation between the participant's Target ratings and their target's Self ratings. Egocentrism refers to how much one anchors their social predictions on selfknowledge. To assess how much a participant engaged in egocentric strategies, we computed the Pearson's correlation between the participant's Target ratings and their own Self ratings. Similarity refers to how much two people's emotion transitions correspond to each other. To measure dyadic similarity within the specific domain of emotion transitions, we computed the Pearson's correlation between a participant's Self ratings and their target's Self ratings. Finally, typicality refers how similar one is to the average person in their social environment. To assess a participant's typicality within the specific domain of emotion transitions, we computed the Pearson's correlation between the participant's Self ratings and the average Self ratings of the sample. We applied Fisher's z-transformation to each of these four, respective, correlation indices.

Personality Measures. Participants completed a series of survey questions that measured their personality in four different domains: attitudes, values, recreational activities, and feelings towards different social groups. To measure social and political attitudes, participants indicated, on a seven-point Likert scale, how much they agreed or disagreed with 11 statements (Bahns et al., 2015, 2017). To measure values, participants indicated, on a seven-point Likert scale, how important 12 values (e.g., independence) were to them as guiding principles (Murray et al., 
2002). To measure recreational activities, participants indicated, on a five-point Likert scale, how frequently they engaged in 15 different activities (e.g., going to the movies, going out to eat). To measure feelings towards different social groups, participants indicated, on a seven-point Likert scale, how warm or cold they felt towards 10 social groups (e.g., children, women).

Participants' responses on the personality measures were used to measure their domain general dyadic similarity and typicality. To assess domain general dyadic similarity, we computed the Euclidean distance (total absolute difference) between a participant's responses and those of their targets across all personality measures. To assess domain general typicality, we computed the Euclidean distance between a participant's responses and the sample average responses across all personality measures. These domain general scores were then reverse coded, such that higher value means more similar or typical, to facilitate interpretation.

\section{Analyses}

In the current study, we aimed to demonstrate the rationality of using the self as a reference point for social predictions. To do so, we conducted three sets of interrelated analyses: (i) egocentrism leads to accurate social predictions: this analysis tested the relation between egocentrism and accuracy (ii) typicality cues people to be egocentric: this analysis tested the relation between participant's typicality and egocentrism (iii) typicality is linked to more accurate prediction at zero acquaintance: this analysis tested the relation between participant typicality and accuracy, as well as whether this relation is mediated by egocentrism and similarity.

For each analysis, we computed the bivariate correlation between variables at the individual level, and then using these scores for correlation and regression analyses across individuals at the sample level. For example, to examine the relation between egocentrism and 
accuracy, we first computed each participants' egocentrism and accuracy scores. We then calculated the correlation between the two scores across participants. One issue this approach faced was that the individual level correlation scores of different constructs can share underlying covariates. Egocentrism scores were calculated by correlating each participant's Self ratings with their Target ratings, whereas accuracy scores were calculated by correlation each participant's Target ratings with their target's Self ratings. The two construct scores shared the participants' Target ratings as a covariate. Such covariate sharing can obscure the true relation between correlation-based construct scores.

As such, our analyses applied an iterative subset-and-bootstrap procedure to eliminate the confounds produced by covariate sharing. In each iteration of this procedure, we first divided the 75 state pairs from the emotion transition task into two equally sized subsets. Each subset was used to estimate one individual level score. For example, to estimate the relation between egocentrism and accuracy, we first divided the 75 items into two sets (a 37-38 split in a single iteration, but equally sized in the long run). For each participant, we obtained their egocentrism scores by correlating their Self ratings and Target ratings on item set 1, and then applying Fisher's z-transformation. We obtained accuracy scores for the same participant by correlating their Self ratings and their target's Self ratings on item set 2, and then applying Fisher's ztransformation. Once these scores were computed, we bootstrapped over participants by repeatedly resampling, with replacement, the same number of participants as the actual sample. For each sample, we calculated the correlation between egocentrism an accuracy scores. These two steps were repeated in a nested fashion to obtain an empirical distribution of the relation of interest. Simulations showed that such a subset-and-bootstrap procedure can effectively address 
the covariate-sharing issue, as well as recover true sample-level correlations (see Supplement for details). All analyses that involved covariate-sharing were conducted using this procedure.

The Accuracy of Egocentrism. We examined whether egocentrism constituted an effective strategy for people to achieve accurate emotion predictions. To do so, we tested the correlation between participants' egocentrism scores and accuracy scores. If anchoring social predictions on self-knowledge indeed aids greater accuracy, we expect that egocentrism and accuracy scores would be positively correlated.

Rational Cues for Egocentrism. Our main analysis examined whether egocentrism might be informed by perceiver's typicality. To do so, we calculated the correlation between egocentrism scores and perceiver typicality scores. If typicality informs the extent to which people anchor their social predictions on self-knowledge, we should see positive correlations between egocentrism and typicality.

We also attempted to replicate previous work showing that egocentrism is tuned to dyadic similarity. To do so, we calculated the correlation between egocentrism and dyadic similarity scores. If dyadic similarity informs the extent to which people employ egocentric strategies, we expected that egocentrism and dyadic similarity should be positively correlated.

Given that dyadic similarity and dyad members' typicality were inherently correlated, we also examined whether typicality exerted unique influences on people's egocentric strategy. We conducted linear regression analyses, using both similarity scores and perceiver typicality scores to predict egocentrism scores. If typicality accounts for egocentrism above and beyond dyadic similarity, we would expect typicality scores to positively predict egocentrism scores after controlling for similarity scores. 
The Accuracy of Rational Egocentrism. We examined whether typicality shaped how accurately people can make emotion predictions. To do so, we calculated the correlation between accuracy scores and perceiver typicality scores. If typicality shapes how accurately one can predict others' emotion transitions at zero acquaintance, we should see positive correlations both between accuracy and perceiver typicality.

Finally, we examined a pathway through which typicality is linked to accuracy. Specifically, we tested the possibility that more typical perceivers achieve greater accuracy because they are more egocentric. In a mediation analysis, we tested whether egocentrism scores accounted for the association between perceiver typicality scores and accuracy scores. If more typical individuals achieve greater accuracy by virtue of relying more on self-knowledge, we expect that egocentrism should mediate the typicality-accuracy link. To ensure the generalizability of this mediation analysis, we conducted a convergent analysis using domain general measures of typicality calculated from the personality measures.

\section{Results}

\section{The Accuracy of Egocentrism}

In our first analysis, we tested whether egocentrism is a rational strategy for social inferences by measuring the relation between egocentrism and accuracy scores. Egocentrism scores were positively associated with accuracy scores $(r(114)=.50,95 \% C I=[.33, .64]$; Figure 1), suggesting that those who anchored their emotion predictions more heavily on selfknowledge also achieved overall greater levels of accuracy about their target. That is, egocentrism was a sound strategy that supported accurate social inferences. 


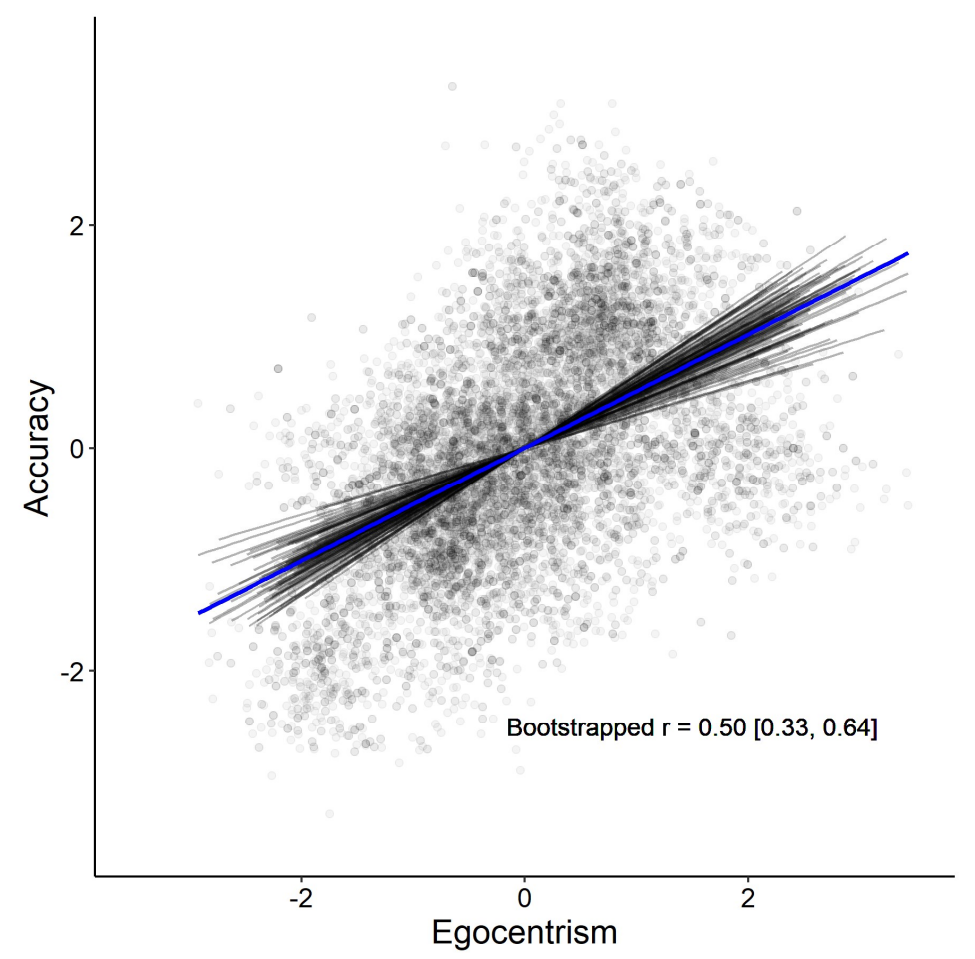

Figure 1. Correlation between Egocentrism and Accuracy scores. People who were more egocentric in their emotion predictions also tended to be more accurate. Points represent 100 randomly selected, normalized samples from the bootstrap analysis. Each black line represents the best fitting regression line for a sample. Blue line represents the mean bootstrapped correlation.

\section{Rational Cues for Egocentrism}

In our second set of analyses, we examined whether people strategically tune their level of egocentrism based on how typical they are of their social surroundings. That is, we examined whether people are more egocentric when it is rational to do so because they are generally more similar to most people. To do so, we tested the correlation between participants' egocentrism scores and typicality scores (Figure 2). We found that typicality positively predicted egocentrism $(r(114)=.60,95 \% C I=[.45, .72])$. We repeated this analyses with domain general typicality, and found the two to be positively correlated $(r(114)=.28,95 \% C I=[.08, .43])$. These results suggest that participants anchored their predictions more heavily on self-knowledge when they were generally more similar to others in their social environment. 

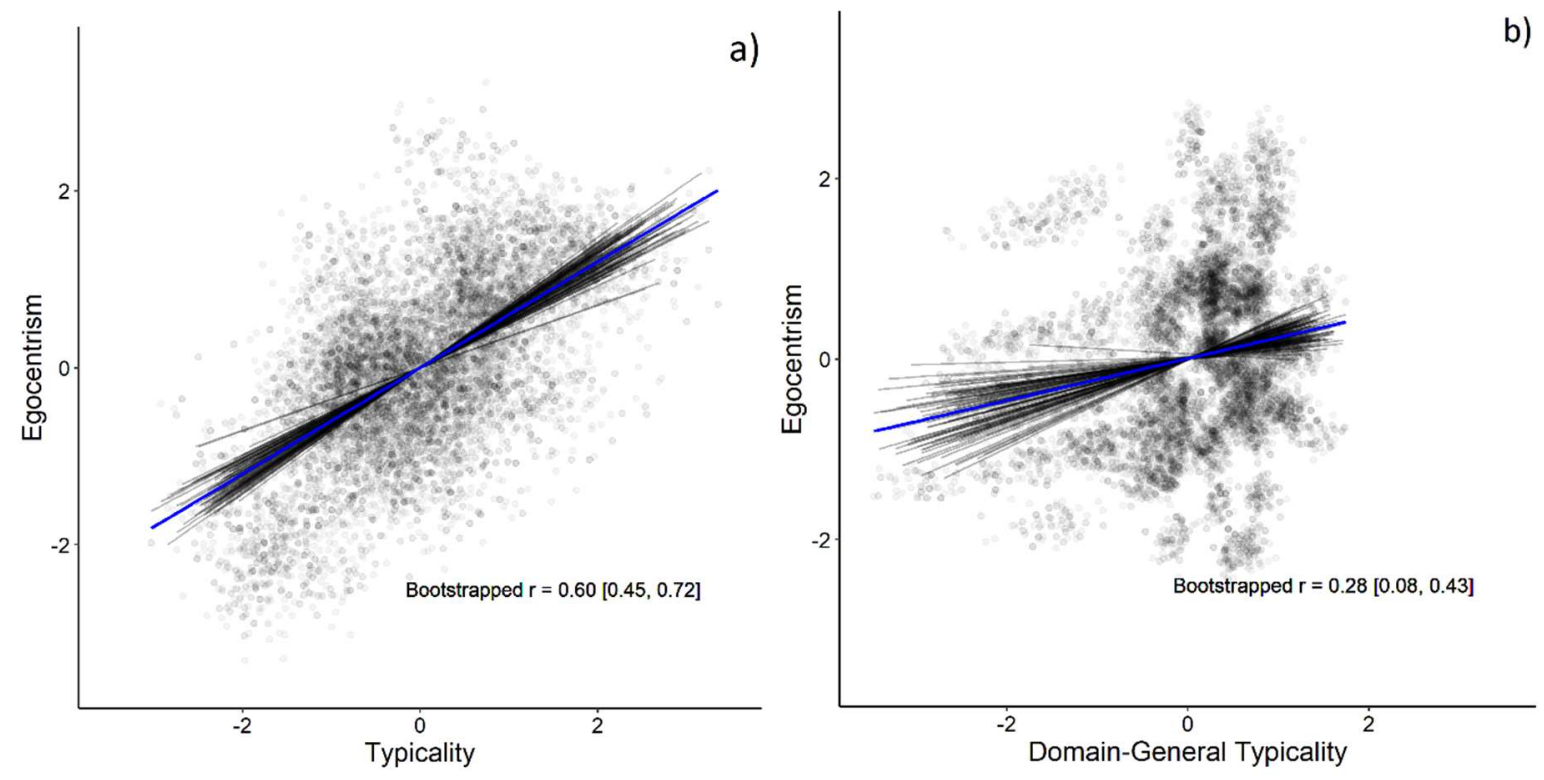

Figure 2. Correlation between Egocentrism and Typicality scores. People who were more typical, both in terms of their (a) emotion dynamics and (b) personality, made more egocentric emotion predictions. Points represent 100 bootstrap samples. Each black line represents the best fitting regression line for a sample. Blue line represents the mean bootstrapped correlation.

In addition, we examined whether people might strategically tune their level of egocentrism based on dyadic similarity. That is, we examined whether people are more egocentric when it is rational to do so because they were more similar to their target. To do so, we tested the correlation between participants' egocentrism scores and dyadic similarity scores. We found that similarity positively predicted egocentrism $(r(114)=.42,95 \% C I=[.25, .58])$. We repeated this analyses with domain general similarity and found it to correlate positively with egocentrism $(r(114)=.21,95 \% C I=[.004, .38])$. These results replicate prior work showing that participants anchored their emotion predictions more heavily on self-knowledge when the target was more similar to themselves.

Both similarity and typicality seem to serve as cues to people to be egocentric in their social inferences about a novel target. We next tested the hypothesis that typicality should serve as a stronger cue than dyadic similarity. In a multiple regression, we used perceiver typicality scores and dyadic similarity scores to simultaneously predict egocentrism scores. As predicted, 
we found that typicality uniquely predicted egocentrism after controlling for similarity $(b=.47$, $95 \% C I=[.26, .66])$, and that similarity did not have a unique effect $(b=.16,95 \% C I=$ $[-.07, .38])$. We repeated this multiple regression using domain general similarity and typicality scores. Consistent with the domain-specific analyses, we found a unique effect of typicality after controlling for similarity $(b=.25,95 \% C I=[.01, .49])$, and no a unique effect for similarity controlling for typicality $(b=.04,95 \% C I=[-.20, .28])$. These findings suggested that egocentric strategies were more strongly cued by typicality than by one's similarity to a specific target.

Taken together, these results supported our hypothesis that people adapt their predictive strategies to contextual cues such as dyadic similarity and typicality, and that typicality might shape predictive strategies to a greater extent than similarity when people make predictions about unfamiliar targets.

\section{The Accuracy of Rational Egocentrism}

In our third set of analyses, we examined whether these cues to be egocentric led typical perceivers to be more accurate in their inference. We started by investigating whether a perceiver's typicality shaped how accurately they could predict a novel target's emotion transitions. To do so, we tested the correlation between participants' accuracy scores and typicality scores. We found that typicality positively predicted accuracy $(r(114)=.43,95 \% C I=$ $[.25, .58])$, suggesting that the more typical a participant was of their social environment, the more accurately they could predict a novel target's emotion transitions. We repeated this analysis with domain general measures of typicality, which were computed using the personality measures. We again found that typicality was positively associated with how accurately participants could predict emotion transitions $(r(114)=.24,95 \% C I=[.03, .42])$. Taken together, 
these results support the hypothesis that one's ability to make accurate emotion predictions in the absence of detailed knowledge about their target was linked to their own typicality within a social context.
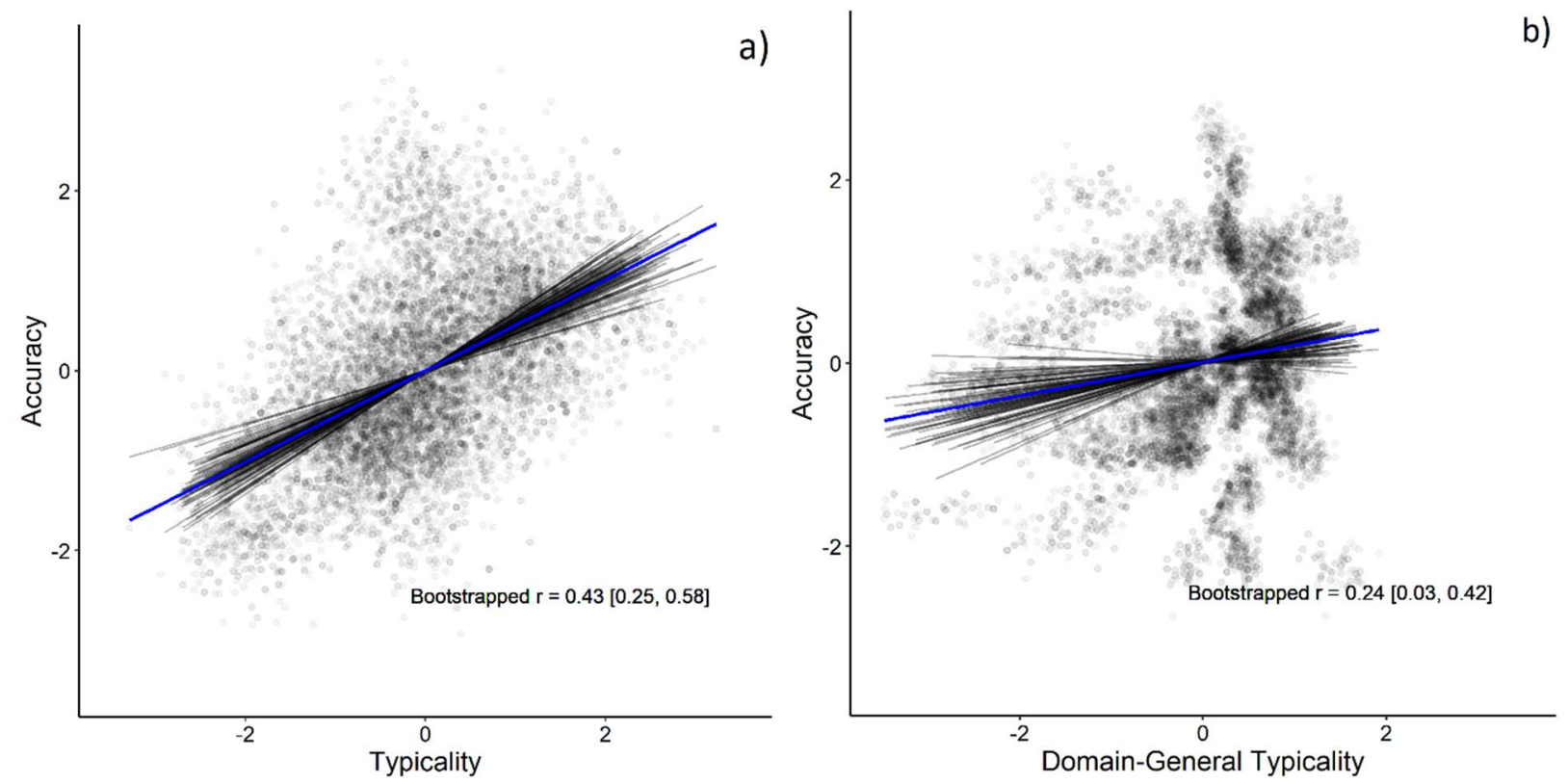

Figure 3. Correlation between Accuracy and both (a) domain-specific and (b) domain-general Typicality scores. People who were more typical, both in terms of their emotion dynamics and their personality, predicted novel targets' emotion transitions more accurately. Points represent 100 bootstrap samples. Each black line represents the best fitting regression line for a sample. Blue line represents the mean bootstrapped correlation.

Next, we aimed to explain why more typical people could make more accurate emotion predictions about novel individuals. To do so, we used mediation models to test the hypothesis that one's typicality leads them to strategically use self-knowledge during social inferences, and that this strategy, in turn, leads to accuracy (Figure 4). In a mediation model, we tested whether egocentrism mediated the relation between typicality and accuracy. The total effect using typicality to predict accuracy was $b=.44(95 \% C I=[.27, .59])$. We detected a positive indirect effect through egocentrism $(b=.21,95 \% C I=[.07, .37])$. The effect of typicality was reduced after mediation, leaving a direct effect of $b=.23(95 \% C I=[-.01, .46])$ and indicating that the association between typicality and accuracy was mediated through egocentrism. This pattern of 
results generalized to domain general analyses, where the total effect of domain general typicality in predicting accuracy was $b=.22(95 \% C I=[.01, .41])$. We detected a positive indirect effect through egocentrism $(b=.12,95 \% C I=[.02, .21])$. The effect of domain general typicality was reduced after mediation, leaving a direct effect of $b=.11(95 \% C I=[-.08, .27])$, suggesting that egocentrism mediated the association between domain general typicality and accuracy. These results further support the idea that the more typical a person is in general the more they might use self-knowledge to make emotion predictions, and thus the more accurate they were.

Typicality can also be associated with accurate emotion predictions through other pathways, such as interpersonal similarity. Typical individuals are more likely to be similar to any given person in their social context. When people make similarly egocentric predictions, those who are more similar to their specific target will be more accurate, In the Supplemental materials, we include an analysis of whether interpersonal similarity accounts for the link between typicality and accuracy in addition to egocentrism.
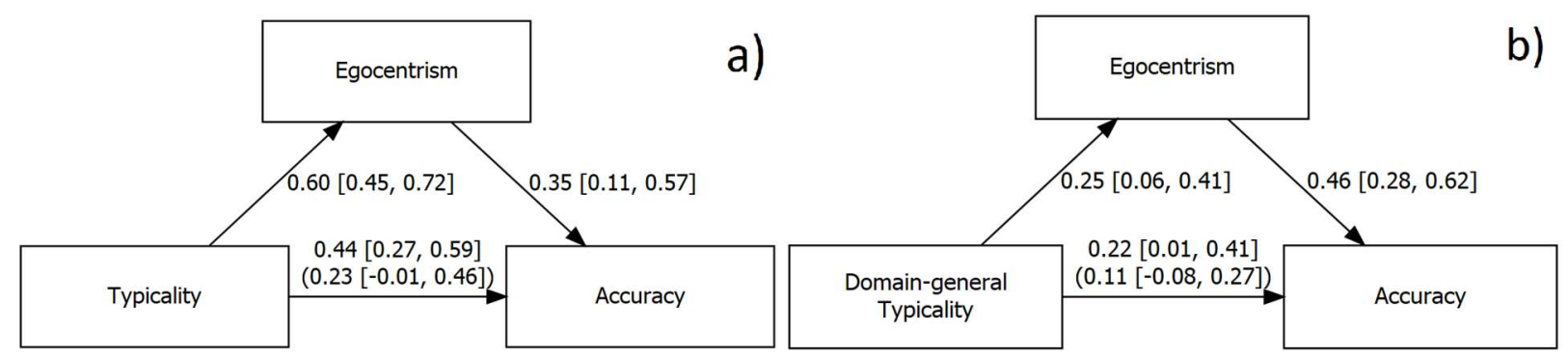

Figure 4. Egocentric inference mediated the Typicality-Accuracy link. Typical perceivers more accurately predicted novel targets' emotion transitions, and they did so by virtue of being more egocentric.

\section{Discussion}

Consistent with our hypotheses, results from this study revealed that an individual's typicality can confer substantial advantages at the outset of new social relationships. More 
typical individuals engage in more egocentric predictions, which in turn makes it more likely that they make accurate inferences about their targets.

\section{Study 2}

In Study 1, we set out to investigate a set of three interrelated ideas. We showed that egocentrism - using knowledge about the self to make inferences about others - could lead to accurate predictions about unfamiliar targets. We then showed that people alter the extent to which they employ an egocentric strategy based on similarity cues, both dyadic similarity and their own global typicality. Finally, we showed that more typical individuals make more accurate prediction about unfamiliar others because they have learned to employ egocentric strategies to a greater extent. The analyses of Study 1 were largely exploratory. In the current study, we aimed to conduct a preregistered replication of these findings in an independent sample, collected using a very similar paradigm.

\section{Methods}

Participants in Study 2 were first-year undergraduate students at Princeton University. Each student signed up for the study with one roommate at the outset of the fall semester. Participants were recruited as the first wave of a second longitudinal study aimed to replicate and extend findings from Study 1. Subsequent waves were not analyzed for the current work. The same sample size of 40 was set a priori and 58 newly formed roommate dyads were recruited ( $N$ $=116,66$ women; Mean age $=18.16, \mathrm{SD}=0.88 ; 41.4 \%$ White, $37.9 \%$ Asian, $12.1 \%$ more than one ethnicity, $4.3 \%$ others, 3.4\% Black or African American, .9\% Native Hawaiian and Pacific Islander; 18\% Hispanic or Latinx). All roommates were assigned by university housing rather than self-selected, and had known each other for 27.5 days on average at the time of study completion (range $=8-81$ days). Two dyads were excluded prior to analyses due to prior 
acquaintanceship (relationship durations $=140$ days and 176 days). All participants provided informed consent in a manner approved by the Princeton University Institutional Review Board.

\section{Procedure}

The measures and procedures used in Study 2 were identical to those in Study 1. Participants completed the emotion prediction task. Their responses were used to compute domain-specific scores of egocentrism, similarity, typicality, and accuracy. Participants’ responses personality measures were again used to compute domain-general versions of those scores. These data were used to conduct a preregistered replication of the three main sets of hypotheses from Study 1: (i) more egocentric emotion predictions will be more accurate, (ii) people who are more typical should make more egocentric predictions, (iii) people who are more typical should make more accurate predictions because they tend to be more egocentric.

\section{Results}

\section{The Accuracy of Egocentrism}

In our first analysis we tested whether egocentrism is a rational strategy, in that it supports accurate social inferences. Results replicated the positive association between participants' egocentrism scores and accuracy scores $(r(110)=.50,95 \% C I=[.31, .65]$; Figure 5). These results further supported the hypothesis that egocentrism can be an effective strategy for social prediction. 


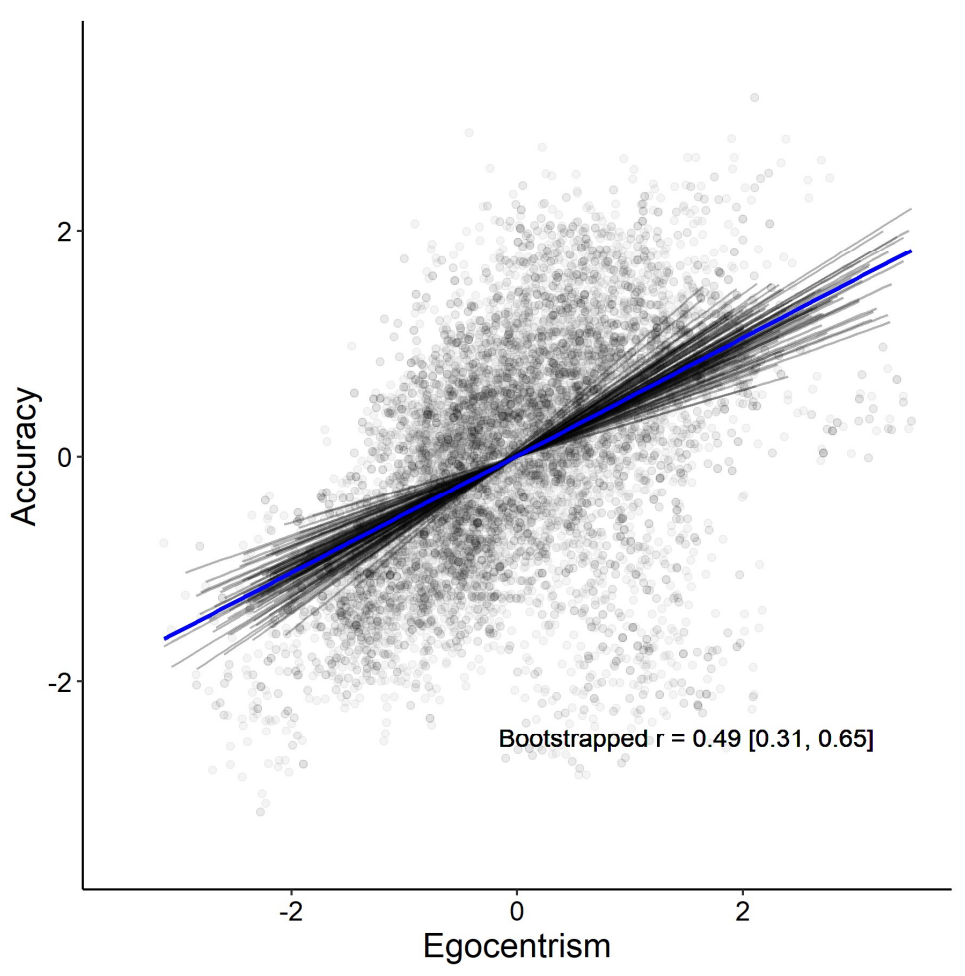

Figure 5. Correlation between Egocentrism and Accuracy scores in the Replication sample. More egocentric perceivers made more accurate emotion predictions. Points represent 100 bootstrap samples. Each black line represents the best fitting regression line for a sample. Blue line represents the mean bootstrapped correlation.

\section{Rational Cues for Egocentrism}

Our second set of analyses tested whether people adjust the use of egocentric strategies based on how typical they are of their social environment. To this end, we tested the correlation between participants' egocentrism scores and typicality scores. The results again fully replicated findings from Study 1 (Figure 6). We found a positive association between typicality scores and egocentrism $(r(110)=.71,95 \% C I=[.58, .81])$. Likewise, domain general typicality positively predicted egocentrism $(r(110)=.41,95 \% C I=[.26, .54])$ 

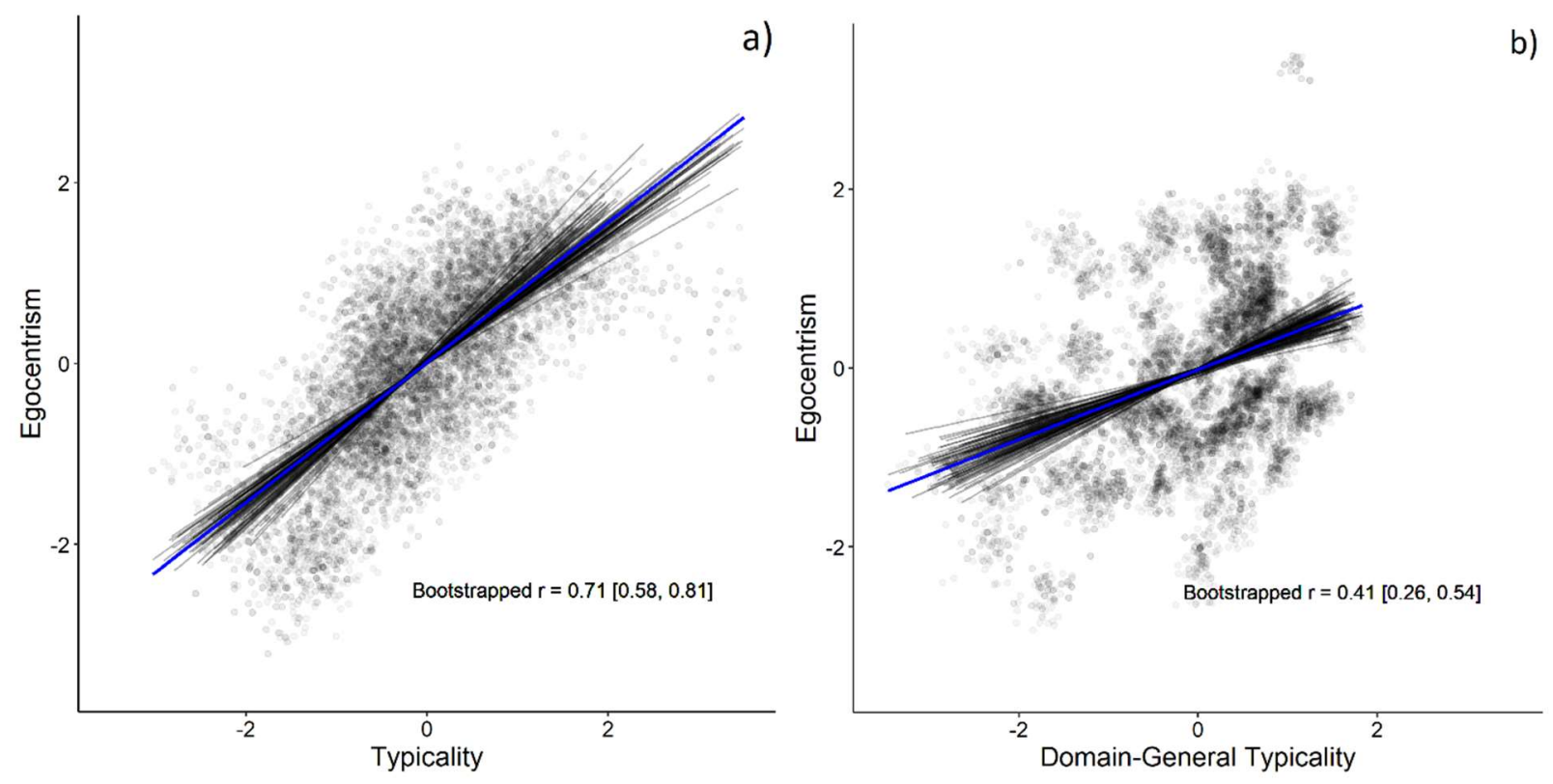

Figure 6. Correlation between Egocentrism and both (a) domain-specific and (b) domain-general Typicality scores from the replication sample. People who were more typical relied more heavily on their self-knowledge to predict others' emotions. Points represent 100 bootstrap samples. Each black line represents the best fitting regression line for a sample. Blue line represents the mean bootstrapped correlation.

We also tested whether egocentric strategies were informed by how similar people were to their specific partner. To do so, we tested the correlation between participants' egocentrism scores and similarity scores. We replicated the positive association between dyadic similarity and egocentrism $(r(110)=.46,95 \% C I=[.28, .62])$. This association extended to domain general similarity, which was also positively associated with egocentrism $(r(110)=.28,95 \% C I=$ $[.09, .44])$. These results show that people tailor their inference strategy based on how relevant self-knowledge is likely to be.

Importantly, we again tested the hypothesis that typicality should more strongly cue egocentrism than dyadic similarity. In a multiple regression, we found that typicality uniquely predicted egocentrism after controlling for similarity $(b=.60,95 \% C I=[.41, .77])$, but similarity did not have a unique effect after controlling for typicality $(b=.11,95 \% C I=$ $[-.08, .31])$. When the regression analysis was repeated using domain general measures, 
typicality again uniquely predicted egocentrism controlling for similarity $(b=.36,95 \% C I=$ $[.17, .54])$, and similarity did not uniquely predict egocentrism after controlling for typicality $(b$ $=.14,95 \% C I=[-.05, .32])$. These findings suggested that egocentric strategies were more strongly cued by one's typicality than by one's similarity to a specific target. These results corroborated Study 1 finding that typicality was a strong cue for egocentric strategies than one's similarity to a specific novel target.

Taken together, this set of findings further supported the idea that people adapt how much they rely on self-knowledge when making predictions about novel individuals. This adjustment tracks more closely with the perceiver's typicality in the social environment than with their similarity to the specific target.

\section{The Accuracy of Rational Egocentrism}

In the third set of analyses, we examined whether typical perceivers were more accurate in their emotion prediction. We first replicated Study 1 findings on the association between typicality and accuracy. We found that a perceiver's typicality was associated with how accurately they predicted a novel individual's emotion transitions. Typicality scores were positively correlated with accuracy scores $(r(110)=.46,95 \% C I=[.28, .61])$. Furthermore, the typicality-accuracy association extended to domain general analyses, where domain general typicality was also positively correlated with accuracy $(r(110)=.37,95 \% C I=[.21, .52])$. Together, these results lent further credence to the idea that more typical individuals could make more accurate emotion prediction in the absence of knowledge about a specific target. 

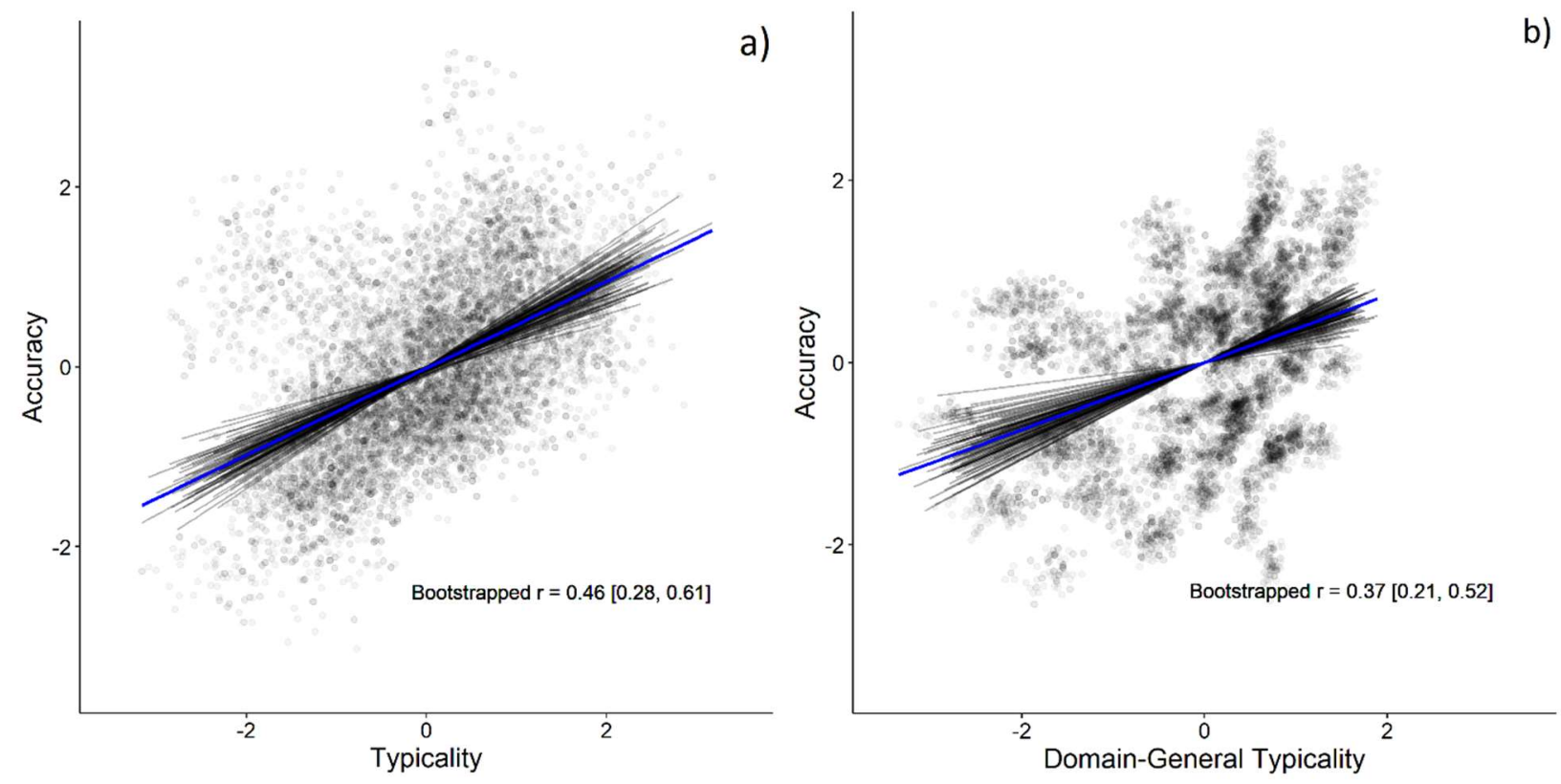

Figure 7. Correlation between Accuracy and both (a) domain-specific and (b) domain-general Typicality scores in the replication sample. Typical perceivers more accurately predicted novel targets' emotion transitions. Points represent 100 bootstrap samples. Each black line represents the best fitting regression line for a sample. Blue line represents the mean bootstrapped correlation.

We then tested the hypothesized pathway that might account for the typicality-accuracy association. More specifically, we replicated Study 1 results showing that typicality influenced accuracy by shaping a person's strategic use of self-knowledge. In a mediation model, egocentrism score partially mediated the association between typicality and accuracy. The total effect of typicality predicant accuracy was $b=.46(95 \% C I=[.27, .64])$. We detected a positive indirect effect through similarity $(b=.23,95 \% C I=[.06, .45])$. After mediation, the effect of typicality was reduced, leaving a direct effect of typicality of $b=.23(95 \% C I=[-.05, .48])$. Likewise, the mediating effect of egocentrism held in domain general analyses. Egocentrism scores mediated the association between domain general typicality and accuracy. The total effect of domain general typicality predicting accuracy was $.33(95 \% C I=[.15, .49])$. We detected a positive indirect effect through egocentrism $(b=.18,95 \% C I=[.07, .30])$. The effect of typicality was reduced to $b=.15(95 \% C I=[-.03, .35])$ after mediation. These replications 
strengthened the conclusion that being more typical of one's environment can aid individuals to make more accurate predictions about others in the absence of person knowledge. They also further supported the idea that more typical individuals achieve more accurate predictions by strategically relying more on self-knowledge to make predictions. Replication analyses on the additional mediating effect of interpersonal similarity can be found in the Supplement.
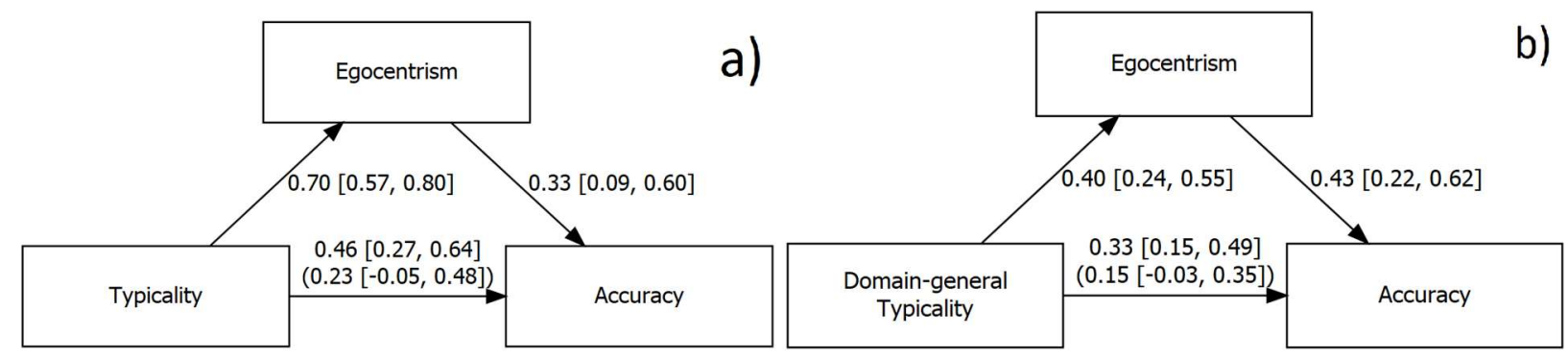

Figure 8. Egocentric inference mediated the Typicality-Accuracy link in the replication sample. Typical perceivers more accurately predicted novel targets' emotion transitions, and they did so by virtue of being more egocentric.

\section{Discussion}

Consistent with our preregistered hypotheses, findings from Study 2 replicated and further supported results from Study 1. People who are typical tend to be more egocentric in their predictions, which can lead them to make more accurate predictions when they encounter novel individuals.

\section{General Discussion}

To create and maintain meaningful interpersonal connections, it is critical that people anticipate others' thoughts and emotions. Imagine meeting up with someone who has recently gone through some stressful events and was quite distraught. How you approach the interaction will depend on how you expect them to be feeling. If you expect them to still be affected, you might try to be a lot more sensitive than if you expect that they have already gone back to their usual self. If this person is an old friend, you might be able to form your expectations based on the kind of person they are or how they've dealt with similar situations in the past. However, 
what if this is someone you've only recently met and had only very limited personal knowledge about? This task of social prediction can be highly challenging, especially in the context of meeting new people, where the perceiver might operate in a vacuum of knowledge about the target. To solve this challenge, people routinely resort to an egocentric strategy, anchoring their social prediction on what they know about themselves. Here we show that egocentrism provides an effective, rational solution to the challenge of social prediction. Across an initial study and a pre-registered replication study, we found robust evidence that egocentric emotion prediction tended to be more accurate. Further, we see that people employ this egocentric strategy when doing so was particularly likely to lead to accurate predictions, such as when they were more typical members of their community.

We found that egocentrism can be an overall effective, rational strategy for social prediction. People who anchored their emotion prediction more on self-knowledge also more accurately predicted an unfamiliar target's emotion transitions. This finding aligns with previous research demonstrating the potential rationality of egocentrism in other domains of social judgment, such as attitudes, beliefs, and preferences (Hoch, 1987; Woo \& Mitchell, 2020). While people often consider egocentrism a mis-calibrated heuristic for thinking about other people, here we show that egocentrism, instead, can be a rational strategy that supports accurate social inferences. Bias is relative. If people have unlimited processing power and all the relevant information about their social target, it would seem ill-advised to make predictions based only on what they know about themselves. However, such an ideal scenario is improbable in the social world, especially during early encounters with a novel social target. Social predictions about a novel target would be a complete shot in the dark if perceivers were not able to draw upon their own self-knowledge. 
We also found that people rationally tune their egocentric strategy, and use selfknowledge to a greater extent when doing so would likely lead to more accurate predictions. More specifically, we found that people's degree of egocentrism tracked social similarity at two levels - dyadic similarity and typicality. People make more egocentric predictions when they are more similar to their target. This dovetails with the extensive literature showing exacerbated egocentric bias for the ingroup (Ames, 2004b; Robbins \& Krueger, 2005). However, here we found that the relation between similarity and egocentrism can be accounted for by typicality. When we observed a positive relation between similarity and egocentrism in the current data, it was because both similarity and accuracy were positively associated with typicality. Furthermore, the tendency for typical perceivers to be more egocentric could be a pathway through which they manage to more accurately predict the emotions of novel, unfamiliar targets. On average, typical perceivers are more likely to be similar to any given person they encounter in their social lives. With repeated feedback from such "chronic similarity," those who are typical of their social context learn, over their lifetime, that their self-knowledge serves as a good proxy for others. This drives them to default more to egocentrism and adapt less to any given dyadic context and serve them particularly well in a highly homogenous community.

On the other hand, those who are less typical might learn to adjust their prediction strategy on a much shorter timescale. They might do so by adapting their predictions in a personspecific manner, particularly at the outset of interacting with a new social partner. However, by attempting to tune their predictions to a greater extent even when their self-knowledge would have sufficed, less typical individuals can end up making less accurate emotion predictions at the outset of interacting with a new social partner. An intriguing possibility is that typicality influences not only how accurately people can make predictions about novel individuals during 
early encounters, but also how their accuracy will change over the time course of interpersonal relationship development. If more typical perceivers have indeed learned to adapt less to each given specific target, they might fail to learn and incorporate useful, person-specific knowledge about their partner, and thus show less improvement in their prediction accuracy over time. In contrast, less typical perceivers might show greater flexibility in encoding and employing targetspecific knowledge in their predictions, and become more accurate over time. Future research should test these predictions by tracking typicality and predictive accuracy over time using longitudinal designs.

Conventionally, rationality has meant acting in accordance with what logic and probability theory dictate. More recently, however, researchers have argued that the rationality of a behavior should be evaluated alongside the ecological contexts in which the decision problem takes place (Marr, 2010). That is, to assess whether people act or make decisions rationally, we must first carefully characterize the computational problem people need to solve, taking into account its natural statistics and the decision maker's resource constraints (Lieder \& Griffiths, 2020). The current studies demonstrated that egocentric strategies provide rational solutions in a naturally occurring, ecologically valid context where emotion prediction occurs, namely a university community.

Nonetheless, the rationality of egocentrism is determined by two characteristics of naturalistic emotion prediction. First, real-life social contexts are often characterized by homophily (McPherson et al., 2001). Through both structural and assortative mechanisms, people that are closely linked in a social network tend to be highly similar. When there is a high level of interpersonal similarity on average, people can make more accurate predictions about others simply by being egocentric. In contrast, when people try to integrate other sources of 
information, they often rely on stereotypical beliefs. Such information could often be unreliable or not rooted in reality, and thus make the predictions less accurate (Eyal et al., 2018). That said, egocentrism might be rational only in a homogenous environment. Both samples tested in the current studies were recruited from a closed university community that has been heavily preselected. Our participants might therefore have been overall more similar to each other than a random sample of people, thus artificially inflating the link between egocentric strategies and prediction accuracy. Future research should aim to explore how social network homogeneity impacts people's social prediction strategies and their efficacy.

Second, it is possible that egocentrism confers advantage on accuracy only in specific domains of social predictions. In the current work, we tested how well people can make predictions about emotion transitions. While people can vary widely in their emotion dynamics, there are overarching, central regularities of how emotions tend to change over time (Cunningham et al., 2013; Thornton \& Tamir, 2017). Due to these latent regularities, people might be more similar to each other in their emotion transitions than in other domains, rendering egocentrism a particularly effective decision strategy for emotion prediction. In other social domains, however, such high levels of interpersonal overlap might not exist. One such example is political beliefs. When there is a wide range of possible beliefs and ideologies and people are highly polarized, it might not be prudent to assume an unfamiliar target will be like the self. Future research on egocentrism in any social domain should aim to characterize the extent of organically occurring interpersonal similarity before drawing conclusions about bias and rationality.

To investigate the relations among typicality, egocentrism, and the accuracy of emotion prediction in an ecologically valid manner, the current work employed a correlational approach. 
While analytical methods such as mediation model and multiple regression can add confidence to the hypothesized causal directions, we ultimately could only draw correlational conclusions. Future research can experimentally manipulate both the homogeneity of the prediction context and the typicality of the perceiver using a paradigm where participants learn about a community of fictional agents. By intervening on the statistical characteristics of the decision problems, we can draw more direct and more fine-grained conclusions about both the efficacy of egocentric prediction, and how people might use such strategies adaptively.

In sum, the current work applies approaches from rational analysis to demonstrate that egocentric strategies are rational, given the natural contexts in which emotion prediction occurs. These results call researchers to reconsider the conventional dichotomy between bias versus rationality. Rather than attempting to classify cognitive phenomena neatly into one of the two categories, future research should consider cognition and its context in a combined, graded manner. Most importantly, these results provided a somewhat paradoxical prescription of how social predictions should be made in a divided world - that sometimes, the best way to step into others' shoes is to try on your own. 


\section{References}

Ames, D. R. (2004a). Inside the Mind Reader's Tool Kit: Projection and Stereotyping in Mental State Inference. Journal of Personality and Social Psychology, 87(3), 340-353.

https://doi.org/10.1037/0022-3514.87.3.340

Ames, D. R. (2004b). Strategies for Social Inference: A Similarity Contingency Model of Projection and Stereotyping in Attribute Prevalence Estimates. Journal of Personality and Social Psychology, 87(5), 573-585. https://doi.org/10.1037/0022-3514.87.5.573

Bahns, A. J., Crandall, C. S., Gillath, O., \& Preacher, K. J. (2017). Similarity in relationships as niche construction: Choice, stability, and influence within dyads in a free choice environment. Journal of Personality and Social Psychology, 112(2), 329-355. https://doi.org/10.1037/pspp0000088

Bahns, A. J., Springer, L. S., \& The, C. (2015). Fostering diverse friendships: The role of beliefs about the value of diversity. Group Processes \& Intergroup Relations.

http://journals.sagepub.com/doi/10.1177/1368430214566893

Bauman, K. P., \& Geher, G. (2002). We think you agree: The detrimental impact of the false consensus effect on behavior. Current Psychology, 21(4), 293-318.

https://doi.org/10.1007/s12144-002-1020-0

Baumeister, R. F., \& Leary. (2017). The Need to Belong: Desire for Interpersonal Attachments as a Fundamental Human Motivation. In Interpersonal Development (pp. 57-89). Routledge. https://doi.org/10.4324/9781351153683-3

Biesanz, J. C., West, S. G., \& Millevoi, A. (2007). What do you learn about someone over time? The relationship between length of acquaintance and consensus and self-other agreement in judgments of personality. Journal of Personality and Social Psychology, 92(1), 119-135. https://doi.org/10.1037/0022-3514.92.1.119

Birch, S. A. J., \& Bloom, P. (2007). The Curse of Knowledge in Reasoning About False Beliefs. Psychological Science, 18(5), 382-386. https://doi.org/10.1111/j.1467-9280.2007.01909.x

Camerer, C., Loewenstein, G., \& Weber, M. (1989). The Curse of Knowledge in Economic Settings: An Experimental Analysis. Journal of Political Economy, 97(5), 1232-1254. https://doi.org/10.1086/261651

Cantor, N., \& Mischel, W. (1979). Prototypicality and personality: Effects on free recall and personality impressions. Journal of Research in Personality, 13(2), 187-205. https://doi.org/10.1016/0092-6566(79)90030-8

Cho, J. C., \& Knowles, E. D. (2013). I'm like you and you're like me: Social projection and selfstereotyping both help explain self-other correspondence. Journal of Personality and Social Psychology, 104(3), 444-456. https://doi.org/10.1037/a0031017 
Clement, R. W., \& Krueger, J. (2002). Social Categorization Moderates Social Projection. Journal of Experimental Social Psychology, 38(3), 219-231.

https://doi.org/10.1006/jesp.2001.1503

Cunningham, W. A., Dunfield, K. A., \& Stillman, P. E. (2013). Emotional States from Affective Dynamics. Emotion Review, 5(4), 344-355. https://doi.org/10.1177/1754073913489749

Dawes, R. M. (1989). Statistical criteria for establishing a truly false consensus effect. Journal of Experimental Social Psychology, 25(1), 1-17. https://doi.org/10.1016/0022-1031(89)90036-X

Eberhardt, J. L., Davies, P. G., Purdie-Vaughns, V. J., \& Johnson, S. L. (2006). Looking Deathworthy: Perceived Stereotypicality of Black Defendants Predicts Capital-Sentencing Outcomes. Psychological Science, 17(5), 383-386. https://doi.org/10.1111/j.14679280.2006.01716.x

Epley, N., Keysar, B., Van Boven, L., \& Gilovich, T. (2004). Perspective Taking as Egocentric Anchoring and Adjustment. Journal of Personality and Social Psychology, 87(3), 327-339. https://doi.org/10.1037/0022-3514.87.3.327

Epskamp, S. (2015). semPlot: Unified visualizations of structural equation models. Structural Equation Modeling, 22(3), 474-483. https://doi.org/10.1080/10705511.2014.937847

Essien, I., Otten, S., \& Degner, J. (2020). Group evaluations as self-group distancing: Ingroup typicality moderates evaluative intergroup bias in stigmatized groups. European Journal of Social Psychology, n/a(n/a). https://doi.org/10.1002/ejsp.2708

Eyal, T., Steffel, M., \& Epley, N. (2018). Perspective mistaking: Accurately understanding the mind of another requires getting perspective, not taking perspective. Journal of Personality and Social Psychology, 114(4), 547-571. https://doi.org/10.1037/pspa0000115

Gilovich, T., Medvec, V. H., \& Savitsky, K. (2000). The spotlight effect in social judgment: An egocentric bias in estimates of the salience of one's own actions and appearance. Journal of Personality and Social Psychology, 78(2), 211-222. https://doi.org/10.1037/0022-3514.78.2.211

Gilovich, T., Savitsky, K., \& Medvec, V. H. (1998). The illusion of transparency: Biased assessments of others' ability to read one's emotional states. Journal of Personality and Social Psychology, 75(2), 332-346. https://doi.org/10.1037/0022-3514.75.2.332

Goldman, L., \& Hogg, M. A. (2016). Going to extremes for one's group: The role of prototypicality and group acceptance. Journal of Applied Social Psychology, 46(9), 544-553. https://doi.org/10.1111/jasp.12382

Granberg, D., Jefferson, N. L., Brent, E. E., \& King, M. (1981). Membership group, reference group, and the attribution of attitudes to groups. Journal of Personality and Social Psychology, 40(5), 833-842. https://doi.org/10.1037/0022-3514.40.5.833

Griffiths, T. L. (2020). Understanding Human Intelligence through Human Limitations. Trends in Cognitive Sciences, 24(11), 873-883. https://doi.org/10.1016/j.tics.2020.09.001 
Griffiths, T. L., Lieder, F., \& Goodman, N. D. (2015). Rational Use of Cognitive Resources: Levels of Analysis Between the Computational and the Algorithmic. Topics in Cognitive Science, 7(2), 217-229. https://doi.org/10.1111/tops.12142

Hoch, S. J. (1987). Perceived consensus and predictive accuracy: The pros and cons of projection. Journal of Personality and Social Psychology, 53(2), 221-234.

https://doi.org/10.1037/0022-3514.53.2.221

Holtz, R. (2003). Intragroup or Intergroup Attitude Projection Can Increase Opinion Certainty: Is There Classism at College? Journal of Applied Social Psychology, 33(9), 1922-1944. https://doi.org/10.1111/j.1559-1816.2003.tb02087.x

Judd, C. M., \& Johnson, J. T. (1981). Attitudes, polarization, and diagnosticity: Exploring the effect of affect. Journal of Personality and Social Psychology, 41(1), 26-36. https://doi.org/10.1037/0022-3514.41.1.26

Keysar, B. (1994). The Illusory Transparency of Intention: Linguistic Perspective Taking in Text. Cognitive Psychology, 26(2), 165-208. https://doi.org/10.1006/cogp.1994.1006

Keysar, Boaz, \& Henly, A. S. (2002). Speakers' Overestimation of Their Effectiveness. Psychological Science, 13(3), 207-212. https://doi.org/10.1111/1467-9280.00439

Li, Y.-Y., \& Hong, Q. (2016). Intergroup Perceptual Accuracy Predicts Real-Life Intergroup Interactions. Group Processes \& Intergroup Relations.

http://journals.sagepub.com/doi/10.1177/1368430201004004004

Lieder, F., \& Griffiths, T. L. (2020). Resource-rational analysis: Understanding human cognition as the optimal use of limited computational resources. Behavioral and Brain Sciences, 43. https://doi.org/10.1017/S0140525X1900061X

Marr, D. (2010). Vision: A Computational Investigation Into the Human Representation and Processing of Visual Information. MIT Press.

McConnell, A. R., Sherman, S. J., \& Hamilton, D. L. (1994). Illusory correlation in the perception of groups: An extension of the distinctiveness-based account. Journal of Personality and Social Psychology, 67(3), 414-429. https://doi.org/10.1037/0022-3514.67.3.414

McPherson, M., Smith-Lovin, L., \& Cook, J. M. (2001). Birds of a Feather: Homophily in Social Networks. Annual Review of Sociology, 27, 415-444.

Murray, S. L., Holmes, J. G., Bellavia, G., Griffin, D. W., \& Dolderman, D. (2002). Kindred spirits? The benefits of egocentrism in close relationships. Journal of Personality and Social Psychology, 82(4), 563-581.

Nosofsky, R. M. (1988). Exemplar-based accounts of relations between classification, recognition, and typicality. Journal of Experimental Psychology: Learning, Memory, and Cognition, 14(4), 700-708. https://doi.org/10.1037/0278-7393.14.4.700 
R core team. (2018). R: A language and environment for statistical computing. $R$ Foundation for Statistical Computing, Vienna, Austria. https://www.R-project.org/

Robbins, J. M., \& Krueger, J. I. (2005). Social Projection to Ingroups and Outgroups: A Review and Meta-Analysis. Personality and Social Psychology Review, 9(1), 32-47.

https://doi.org/10.1207/s15327957pspr0901_3

Rosch, E., Simpson, C., \& Miller, R. S. (1976). Structural bases of typicality effects. Journal of Experimental Psychology: Human Perception and Performance, 2(4), 491-502.

https://doi.org/10.1037/0096-1523.2.4.491

Rosenbaum, M. E. (1986). The repulsion hypothesis: On the nondevelopment of relationships. Journal of Personality and Social Psychology, 51(6), 1156-1166. https://doi.org/10.1037/00223514.51.6.1156

Ross, L., Greene, D., \& House, P. (1977). The "false consensus effect": An egocentric bias in social perception and attribution processes. Journal of Experimental Social Psychology, 13(3), 279-301. https://doi.org/10.1016/0022-1031(77)90049-X

Rosseel, Y. (2012). lavaan: An R Package for Structural Equation Modeling. Journal of Statistical Software, 48(1), 1-36. https://doi.org/10.18637/jss.v048.i02

Stinson, L., \& Ickes, W. (1992). Empathic accuracy in the interactions of male friends versus male strangers. Journal of Personality and Social Psychology, 62(5), 787-797.

https://doi.org/10.1037/0022-3514.62.5.787

Tajfel, H. (1970). Experiments in Intergroup Discrimination. Scientific American, 223(5), 96103.

Tamir, D. I., \& Mitchell, J. P. (2013). Anchoring and adjustment during social inferences. Journal of Experimental Psychology: General, 142(1), 151-162.

https://doi.org/10.1037/a0028232

Tamir, D. I., \& Thornton, M. A. (2018). Modeling the Predictive Social Mind. Trends in Cognitive Sciences, 22(3), 201-212. https://doi.org/10.1016/j.tics.2017.12.005

Terracciano, A., Abdel-Khalek, A. M., Ádám, N., Adamovová, L., Ahn, C. -k., Ahn, H. -n., Alansari, B. M., Alcalay, L., Allik, J., Angleitner, A., Avia, A., Ayearst, L. E., Barbaranelli, C., Beer, A., Borg-Cunen, M. A., Bratko, D., Brunner-Sciarra, M., Budzinski, L., Camart, N., ... McCrae, R. R. (2005). National Character Does Not Reflect Mean Personality Trait Levels in 49 Cultures. Science (New York, N.Y.), 310(5745), 96-100. https://doi.org/10.1126/science.1117199

Thornton, M. A., \& Tamir, D. I. (2017). Mental models accurately predict emotion transitions. Proceedings of the National Academy of Sciences, 114(23), 5982-5987.

https://doi.org/10.1073/pnas.1616056114

Wojcieszak, M. (2008). False Consensus Goes Online: Impact of Ideologically Homogeneous Groups on False Consensus. The Public Opinion Quarterly, 72(4), 781-791. 
Woo, B. M., \& Mitchell, J. P. (2020). Simulation: A strategy for mindreading similar but not dissimilar others? Journal of Experimental Social Psychology, 90, 104000.

https://doi.org/10.1016/j.jesp.2020.104000

Zhao, Z., Thornton, M. A., \& Tamir, D. I. (2020). Accurate emotion prediction in dyads and groups and its potential social benefits. Emotion. https://doi.org/10.1037/emo0000890 